Schwerpunkt

Kardiologe 2021 · 15:92-100

https://doi.org/10.1007/s12181-021-00461-0

Angenommen: 28. Januar 2021

Online publiziert: 10. März 2021

(c) Deutsche Gesellschaft für Kardiologie -

Herz- und Kreislaufforschung e.V. Published by

Springer Medizin Verlag GmbH, ein Teil von

Springer Nature - all rights reserved 2021

\author{
Nikos Werner ${ }^{1} \cdot$ Franz-Josef Neumann ${ }^{2}$ \\ 'Herzzentrum Trier, Innere Medizin III - Kardiologie und Intensivmedizin, Krankenhaus der Barmherzigen \\ Brüder Trier, Trier, Deutschland \\ ${ }^{2}$ Klinik für Kardiologie und Angiologie II, Universitäts-Herzzentrum Freiburg · Bad Krozingen, Bad \\ Krozingen, Deutschland
}

\title{
Entwicklung kardiovaskuläre Medizin 2020
}

\author{
Ischämische Herzerkrankungen
}

\section{Chronisches Koronarsyndrom}

Das Jahr 2020 wird auch kardiologisch ein erinnerungswürdiges Jahr bleiben. Der Zusammenhang zwischen COVID19 und chronischem (CCS) bzw. akutem Koronarsyndrom (ACS) hat uns bereits seit Beginn der Pandemie beschäftigt. Schwierig gestaltet sich nach wie vor die Diagnostik eines CCS in Zeiten knapper Ressourcen durch die Pandemie. Hier wird in den gegenwärtigen ESC(European Society of Cardiology)Leitlinien [1] die ärztliche Entscheidungsfreiheit, angepasst an die lokale COVID-Infektionslage, in den Vordergrund gestellt: Wo immer möglich, sollte die verfügbare und klinisch passende Diagnostik eingesetzt werden (cave: Stressuntersuchungen mit engem PatientenPersonal-Kontakt) mit der Einschränkung, dass Patienten mit vermuteter ausgeprägter Ischämie bevorzugt untersucht werden sollten. Der Patient mit diagnostiziertem CCS braucht eine umfassende, nichtmedikamentöse und medikamentöse Begleittherapie, die in den entsprechenden Leitlinien aus dem Jahr 2019 ausführlich dargelegt wird und an das ischämische Risiko des Patienten angepasst ist. Die Qual der Wahl, ob rein medikamentös, interventionell oder chirurgisch behandelt werden muss, wird beim CCS allerdings nicht erst seit COVID-19 kontrovers diskutiert. In 2020 haben uns die Ergebnisse der ISCHEMIA-Studie [2], die den prognostischen Nutzen der invasiven Diagnostik mit folgender Revaskularisation gegen- über einer alleinigen optimalen medikamentösen Therapie (OMT) bei Patienten mit stabiler koronarer Herzerkrankung (KHK) untersucht hat, weiter beschäftigt [2]. Insgesamt wurden in der 2020 im NEJM publizierten Studie mehr als 5000 Patienten eingeschlossen und 1:1 randomisiert. Primärer Endpunkt der ISCHEMIA-Studie war die Kombination aus kardialem Tod, Myokardinfarkt, Hospitalisierung infolge instabiler Angina pectoris, Herzinsuffizienz oder Reanimation nach Herzstillstand. Als sekundäre Endpunkte wurden die Dauer von der Randomisierung bis zum Auftreten von kardialen Ereignissen (kardiovaskulärer Tod und Myokardinfarkt) und eine Verbesserung der Lebensqualität definiert. Nach einem medianen Beobachtungszeitraum von 3,3 Jahren konnte keine Prognoseverbesserung durch das invasive Vorgehen gegenüber einem konservativen Vorgehen nachgewiesen werden.

In der Gruppe der Patienten mit einer invasiven Strategie vs. alleinige OMT konnte eine Verbesserung der Lebensqualität und Reduktion der Anginapectoris-Symptomatik (Risikoreduktion $30 \%$, NNT ["number needed to treat"] =3) nachgewiesen werden. Eine Verbesserung der Symptomatik konnte v. a. bei hochsymptomatischen Patienten mit hoher Anginafrequenz nachgewiesen werden (mehrmals wöchentlich oder mehrmals im Monat).

Spezifische Aspekte der ISCHEMIAStudie beschäftigen uns weiterhin noch: So wurden Patienten mit relevanter
Hauptstammstenose, einem akuten Myokardinfarkt innerhalb der letzten 2 Monate vor Studieneinschluss, einer Herzinsuffizienz mit reduzierter LV(linksventrikulär)-Funktion oder starker Angina pectoris trotz OMT nicht eingeschlossen. Hier kam der KoronarCT (Computertomographie) eine bedeutende Rolle zu: Alle Patienten mit deutlichem Ischämienachweis (in der Studie eigentlich definiert als $>10 \%$ des linken Ventrikels, jedoch $25 \%$ nur Belastungs-EKG [Elektrokardiogramm]) erhielten vor Einschluss eine CT zum Ausschluss einer Hauptstammstenose! Dies bedeutet in der klinischen Praxis, dass wir bei Patienten mit Ischämienachweis zumindest eine Hauptstammstenose ausschließen müssen.

\section{》) Die invasive Strategie verbesserte die Lebensqualität und reduzierte die Angina- pectoris-Symptomatik}

Weiterer Aspekte der Studie wie die hohe Cross-over-Rate (insgesamt $20 \%$ der Patienten, die in die invasive Gruppe randomisiert wurden, erhielten keine PCI [perkutane koronare Intervention] oder Bypassoperation; $21 \%$ der konservativ behandelten Patienten erhielten im Verlauf eine invasive Revaskularisation) wurden bereits an anderer Stelle ausführlich diskutiert.

Interessant ist in diesem Zusammenhang auch die detaillierte Analyse der 
Hier steht eine Anzeige.

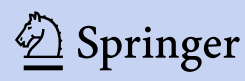


Endpunkte: Myokardinfarkte waren der relevante Treiber der Endpunkte innerhalb des primären und sekundären Endpunkts. Die unterschiedliche Definition von Myokardinfarkt anhand von Biomarkern innerhalb der ISCHEMIAStudie hat relevanten Einfluss auf die Ereignisrate und die Endpunkte. So führte die primäre Myokardinfarktdefinition (basierend auf CK[Kreatinkinase]MB[Muscle-Brain type]-Konzentrationen) $\mathrm{zu}$ vergleichbaren primären und sekundären Endpunkten zwischen beiden Therapiegruppen, während die sekundäre Infarktdefinition (basierend auf Troponin-Konzentrationen) zu mehr Ereignissen in der invasiven Therapiegruppe führte [3]. Dagegen steht die Beobachtung, dass im Gegensatz zu prozeduralen Infarkten Typ-1-Myokardinfarkte eine klare Korrelation mit einem erhöhten Risiko zu versterben hatten und diese signifikant reduziert waren in der invasiven Gruppe im Vergleich zur medikamentösen Therapie.

Zusammenfassend ließ sich im Kollektiv der ISCHEMIA-Studie anhand des primären Endpunkts kein prognostischer Vorteil der Myokardrevaskularisation nachweisen. Es zeigte sich jedoch eine signifikante und klinisch bedeutsame Verminderung spontaner Myokardinfarktinfarkte (MI) im Langzeitverlauf, die mit einer erhöhten Rate periinterventioneller MI erkauft wurde. Während die periinterventionellen MI mit einer nur geringen und statistisch nicht signifikanten Erhöhung der Sterblichkeit einhergingen, war die Sterblichkeit nach spontanen Infarkten erheblich und statistisch signifikant erhöht. Das Risikoprofil und die Zahl der in die ISCHEMIAStudie eingeschlossenen Patienten sowie die Dauer der Nachverfolgung reichen nicht aus, um zu zeigen, dass das verminderte Risikos von spontanen Infarkten im Arm mit Myokardrevaskularisation im Vergleich zum konservativen Arm zu einem bessern Überleben führt. Zusätzlich zur Verminderung des postinterventionellen Myokardinfarktrisikos ergab die angestrebte Myokardrevaskularisation eine statistisch signifikante und klinisch bedeutsame Verminderung der Angina pectoris sowie eine Verbesserung der Lebensqualität im Vergleich zur konservativen Behandlungsstrategie. Die Bedeutung einer optimalen medikamentösen Behandlung des chronischen Koronarsyndroms unabhängig von der Revaskularisationstherapie bleibt hiervon unberührt.

\section{Akutes Koronarsyndrom}

Größere Studien, die zu einem Paradigmenwechsel auf dem Gebiet der akuten Koronarsyndrome (ACS) geführt hätten, wurden im Jahr 2020 nicht publiziert. Dennoch gab es eine Reihe neuer Erkenntnisse, unter anderem auch aus Subanalysen zuvor publizierter randomisierter Studien. Ein Meilenstein war die Präsentation der neuen Leitlinien der European Society of Cardiology (ESC) zu ACS ohne ST-Streckenhebung (NSTEACS) [4]. Wichtige Publikationen aus Jahr 2020 führen die dort vorgestellten Konzepte weiter.

\section{Diagnostik, Behandlungsstrate- gie und Risikostratifizierung}

Diagnostik. Nach Ausschluss von STStreckenhebungen ist die Bestimmung des hochsensitiven Troponins von zentraler Bedeutung für die Diagnostik des ACS. Neben dem etablierten 0/1-h-Algorithmus empfiehlt die neue Leitlinie erstmals auch gleichberechtigt einen 0/2h-Algorithmus [4].

Behandlungsstrategie. Für Patienten mit akutem Koronarsyndrom ohne STStreckenhebung wird bei mittlerem bis hohem Risiko weiterhin die invasive Behandlungsstrategie favorisiert [4]. Dies wird durch Arbeiten gestützt, die 2020 neu erschienen sind. So konnte die SENIOR-NSTEMI-Studie zeigen, dass auch bei Patienten mit einem Alter von $\geq 80$ Jahren die invasive Strategie mit einem Überlebensvorteil verbunden ist [5]. Auch zeigte eine neuere Metaanalyse, dass bei Patienten mit ACS die perkutane Koronarintervention (PCI) das Risiko von Tod und Myokardinfarkt reduziert [6].

Nur bei Patienten mit niedrigem Risiko ist ein "selektiv-invasives“ Vorgehen angezeigt [4]. Dabei wird einer möglichen invasiven Koronarangiographie entweder ein bildgebender Funktionstest oder eine kardiale Computertomographie-Angiographie (CCTA) vorgeschaltet. Analog zu der bereits 2019 erschienenen Leitlinie zu chronischen Koronarsyndromen wertet die neue Leitlinie die Bedeutung der CCTA auf. Dies wird durch die 2020 erschienene publizierte VERDICT(Very Early Versus Deferred Invasive Evaluation Using Computerized Tomography in Patients With Acute Coronary Syndromes)-Studie gestützt, bei der in einer ergänzenden Beobachtungsstudie eine verblindete CCTA durchgeführt wurde [7]. In dieser Studie hatte die CCTA eine hohe diagnostische Genauigkeit bezüglich des Ausschlusses einer klinisch relevanten koronaren Herzkrankheit bei Patienten mit NSTEACS.

\section{) Nur bei Patienten mit niedrigem Risiko ist ein „selektiv- invasives" Vorgehen angezeigt}

Eine weitere Ausnahme von der frühinvasiven Strategie stellen hämodynamisch stabile Patienten ohne ST-Streckenhebung nach präklinischer Reanimation wegen Kammerflimmerns dar. Hier ist eine verzögerte Angiographie anstelle der sofortigen Angiographie angeraten. Dies wird durch das 2020 publizierte 1-Jahres-Ergebnis der COACT(Coronary Angiography after Cardiac Arrest)-Studie gestützt, das zeigt, dass eine frühe Koronarangiographie keine Vorteile bringt gegenüber einer verzögerten Angiographie nach neurologischer Erholung der Patienten [8].

Risikostratifizierung. Bei Patienten mit NSTE-ACS und hohem Risiko sollten die Patienten weiterhin innerhalb von $24 \mathrm{~h}$ einer invasiven Diagnostik zugeführt werden. Kriterien für ein solches hohes Risiko sind die Diagnose eines Myokardinfarkts ohne ST-Streckenhebung (NSTEMI), dynamische oder vermutlich neue ST-/T-Segment-Veränderungen vereinbar mit Myokardischämie, transiente ST-Streckenhebungen sowie ein GRACE(Global Registry of Acute Coronary Events)-Risk-Score von über 140 [4]. Durch eine 2020 erschienene Arbeit 
wird die Bedeutung des GRACE-RiskScores relativiert. Diese Arbeit verglich randomisiert die routinemäßige Verwendung des GRACE-Risk-Scores mit klinischer Einschätzung ohne Score-Berechnung [9]. Mit 2318 eingeschlossenen Patienten ergab sich hierbei kein Vorteil der routinemäßigen Verwendung des GRACE-Risk-Scores in Bezug auf den primären kombinierten Endpunkt von Tod und Myokardinfarkt im 1-JahresVerlauf.

\section{Myokardrevaskularisation}

Komplette Revaskularisation. Bei Patienten mit NSTE-ACS oder Myokardinfarkt mit ST-Streckenhebung (STEMI) und Mehrgefäßerkrankung sollte eine komplette Revaskularisation erfolgen. Hierzu sind bereits in den letzten Jahren mehrere Studien erschienen, zuletzt die große COMPLETE(Complete versus Culprit-Only Revascularization Strategies to Treat Multivessel Disease after Early PCI for STEMI)-Studie. Diese Studien wurden 2020 in 2 Metaanalysen zusammengefasst, die beide zeigen, dass bei Patienten mit STEMI und Mehrgefäßerkrankung die komplette Revaskularisation im Vergleich zur alleinigen PCI der Culprit-Läsion die kardiovaskuläre Sterblichkeit sowie das Risiko von Myokardinfarkt und erneuter Revaskularisation vermindert $[10,11]$. Eine dieser Metaanalysen untersuchte auch die mögliche Rolle von funktionellen Messungen mittels „fractional flow reserve“ (FFR) zur Auswahl der Interventionsziele. Diese Metaanalyse fand keine Heterogenität im Behandlungseffekt der kompletten Revaskularisation zwischen Studien mit und ohne FFRFührung. Dieser Befund muss jedoch vorsichtig interpretiert werden, da bisher kein direkter Vergleich zwischen FFR- und rein angiographisch geführter PCI im akuten Myokardinfarkt vorliegt. Eine Subanalyse der COMPLETEStudie ergab, dass sich der Vorteil der kompletten Revaskularisation v. a. aus der Behandlung von Stenosen mit einer Lumeneinengung von $\geq 60 \%$ bei der quantitativen Koronarangiographie ergab [12]. Die neue ESC-Leitlinie zur NSTE-ACS empfiehlt nun auch bei $\mathrm{Pa}$ -

Kardiologe 2021 · 15:92-100 https://doi.org/10.1007/s12181-021-00461-0

○) Deutsche Gesellschaft für Kardiologie - Herz- und Kreislaufforschung e.V. Published by Springer

Medizin Verlag GmbH, ein Teil von Springer Nature - all rights reserved 2021

\section{N. Werner · F.-J. Neumann}

\section{Entwicklung kardiovaskuläre Medizin 2020. Ischämische Herzerkrankungen}

\section{Zusammenfassung}

Auf dem Gebiet der ischämischen Herzerkrankungen war 2020 ein spannendes Jahr. Die COVID-19-Pandemie hat Diagnostik und Therapie der koronaren Herzkrankheit in vielen Bereichen stark beeinflusst. Daneben haben die Ergebnisse der ISCHEMIA-

Studie zumindest in Teilen die bisherige Praxis in der Behandlung des stabilen, chronischen Koronarsyndroms (CCS) infrage gestellt. In dieser Studie konnte keine Prognoseverbesserung durch Myokardrevaskularisation bei Patienten mit stabiler Ischämie nachgewiesen werden, wohl aber eine Verbesserung von Lebensqualität und Angina-pectoris-Beschwerden. Darüber hinaus wurden neue europäische Leitlinien zum ACS (akutes Koronarsyndrom) ohne ST-Streckenhebung (NSTE-ACS) vorgestellt. Wichtige Publikationen aus dem Jahr 2020 führen die dort vorgestellten Konzepte weiter. So konnte der Nutzen der frühinvasiven
Behandlung beim NSTE-ACS erstmals auch für die $\geq 80$-Jährigen nachgewiesen werden. Neuere Analysen bestätigen ferner das Konzept der kompletten Revaskularisation bei ST-Hebungsinfarkt ohne Schock und Mehrgefäßerkrankung. Dieses Konzept wird nun auf das NSTE-ACS übertragen. In der antithrombozytären Therapie stellen aktuelle Studien die antithrombozytäre Vorbehandlung als Teil der frühinvasiven Strategie bei unbekanntem Koronarstatus infrage. Mehrere Studien zeigen den potenziellen Nutzen einer Deeskalation der antithrombozytären Therapie nach NSTE-ACS insbesondere bei älteren Patienten.

Schlüsselwörter

Chronisches Koronarsyndrom · Akutes Koronarsyndrom · Myokardinfarkt - Thrombozytenaggregationshemmer . Perkutane Koronarintervention

\section{Advances in cardiovascular medicine 2020. Ischemic heart diseases}

\section{Abstract}

In the field of ischemic heart disease 2020 was an exciting year. The coronavirus disease 2019 (COVID-19) pandemic had a major impact on the diagnostics and treatment of coronary heart disease in many ways. In addition, the results of the ISCHEMIA study have at least in part questioned the previous practice of the treatment of stable chronic coronary syndrome (CCS). In this study, myocardial revascularization was not associated with improvement in the prognosis of patients with stable ischemia but resulted in an improvement in the quality of life and angina pectoris symptoms compared to optimal medical therapy. In the field of acute coronary syndromes (ACS), publications from 2020 enhanced the concepts of the new European guidelines on non-ST-segment elevation ACS (NSTE-ACS). For the first time, the benefit of early invasive treatment in NSTE-ACS could be demonstrated for patients $\geq 80$ years of age. Recent analyses also confirmed the concept of complete revascularization for multivessel disease in ST-segment elevation myocardial infarction without shock. This concept is now being applied to NSTEACS. In antiplatelet therapy, recent studies challenged antiplatelet pretreatment as part of the early invasive strategy with unknown coronary artery status. Several studies showed the potential benefit of deescalation of antiplatelet therapy after NSTEACS, especially in older patients.

\section{Keywords}

Chronic coronary syndrome - Acute coronary syndrome $\cdot$ Myocardial infarction · Antiplatelet therapy - Percutaneous coronary intervention tienten mit NSTE-ACS eine komplette Revaskularisation, die im Rahmen des primären Eingriffs oder kurzfristig im Intervall durchgeführt werden kann.
Kardiogener Schock. Eine Ausnahme von der Empfehlung zur frühen kompletten Revaskularisation bei ACS stellen die Patienten mit kardiogenem Schock bei akutem Myokardinfarkt dar. Dies hatte bereits die CULPRIT-SHOCK(Culprit 
Lesion Only PCI versus Multivessel PCI in Cardiogenic Shock)-Studie gezeigt, und die darauf basierenden Empfehlungen sind bereits in früheren Leitlinien verankert. Eine 2020 publizierte Registerstudie mit 64.301 Patienten bestärkt dies noch einmal, indem sie zeigt, dass die Mehrgefäß-PCI im kardiogenen Schock bei Infarkt mit einem ungünstigen Ergebnis für die Patienten einhergeht [13].

Stent-Design. Bezüglich des Stent-Designs gab es die Vermutung, dass bei Patienten mit ACS Drug-eluting-Stents (DES) mit bioresorbierbarem Polymer DES mit permanentem Polymer überlegen sein könnten. Diese Frage wurde in der 2020 publizierten HOST-REDUCEPOLYTECH-ACS(Harmonizing Optimal Strategy for Treatment of coronary artery diseases - comparison of REDUCtion of prasugrEl dose or POLYmer TECHnology in ACS patients)-Studie untersucht [14]. Hierbei ergab sich, dass im 1-Jahres-Verlauf der primäre Endpunkt von Tod, Myokardinfarkt und wiederholter Revaskularisation zwischen beiden Stenttypen nicht signifikant unterschiedlich war. Für die „target vessel failure“ (kardialer Tod, Zielgefäßmyokardinfarkt und Zielgefäßrevaskularisation) ergaben die DES mit permanentem Polymer sogar ein gering, aber signifikant besseres Ergebnis als DES mit bioresorbierbarem Polymer. Insgesamt fand sich somit kein Argument für die präferenzielle Verwendung von DES mit bioresorbierbarem Polymer bei Patienten mit ACS.

\section{Antithrombotische Therapie}

Vorbehandlung. Auf der Basis von Studien und Metaanalysen, die bereits in den vergangenen Jahren erschienen sind, gibt die neue ESC-Leitlinie zu NSTEACS erstmals die generelle Empfehlung, bei Patienten mit NSTE-ACS und unbekannter Koronaranatomie auf eine Vorbehandlung mit einem $\mathrm{P}_{2} \mathrm{Y}_{12}$-Inhibitor zu verzichten, wenn eine frühinvasive $\mathrm{Be}$ handlung geplant ist. Diese Empfehlung wird durch eine 2020 erschienene Subanalyse der ISAR-REACT(Intracoronary Stenting and Antithrombotic Regimen
Rapid Early Action for Coronary Treatment)-5-Studie gestützt. Diese konnte zeigen, dass bei Patienten mit NSTEACS eine Vorbehandlung mit Ticagrelor im Vergleich zur Gabe von Prasugrel im Katheterlabor keinen erkennbaren Vorteil in Bezug auf die 30-Tage-Inzidenz von Tod, Myokardinfarkt und Schlaganfall bringt [15].

Wahl des $\mathbf{P 2}_{\mathbf{1 2}}$-Inhibitors. Eine Reihe von Studien, die 2020 erschienen sind, beschäftigt sich mit der Frage der optimalen Wahl des P2Y $\mathrm{Y}_{12}$-Inhibitors bei Patienten mit NSTE-ACS. Aufgrund der ISARREACT-5-Studie sieht die neue Leitlinie hier erstmals eine Präferenz für Prasugrel im Vergleich zu Ticagrelor bei Patienten, die mit PCI behandelt werden. Dies wird durch eine Subanalyse der ISAR-REACT-5-Studie für das NSTEACS-Kollektiv gestützt [12]. Vergleichbare Ergebnisse wurden auch für die präspezifizierte Subgruppe der Patienten mit STEMI gefunden [16].

Zwei 2020 erschienene Kohortenstudien, eine aus Kanada, eine aus Korea, beschäftigen sich mit dem Vergleich zwischen Ticagrelor und Clopidogrel bei Patienten mit akutem Koronarsyndrom, die sich einer PCI unterziehen. In der kanadischen Studie war die Verwendung von Ticagrelor im Vergleich zu Clopidogrel nicht mit einer statistisch signifikanten Reduktion des kombinierten Endpunkts von Gesamtsterblichkeit, Hospitalisation wegen ACS, ungeplanter koronarer Revaskularisation und Stentthrombose im 1-Jahres-Verlauf verbunden, es fand sich jedoch eine höhere Inzidenz von Blutungskomplikationen und Dyspnoe unter Ticagrelor [17]. Auch in der koreanischen Studie war nach „propensity score matching“ der Nettoeffekt bezüglich ischämischer und hämorrhagischer Ereignisse nach 12 Monaten neutral [18]. Beide Studien müssen aufgrund der Limitationen von Kohortenstudien vorsichtig interpretiert werden.

Von besonderem Interesse sind ältere Patienten, bei denen das Blutungsrisiko besonders hoch ist. Hierzu wurde die randomisierte POPular-AGE-Studie 2020 publiziert [19]. In dieser Studie wurden Patienten mit einem Mindestalter von 70 Jahren zusätzlich zur
Standardtherapie randomisiert entweder einer antithrombozytären Therapie mit Clopidogrel oder einem der potenteren P2Y 12 -Inhibitoren, Ticagrelor (zu 95\%) oder Prasugrel, zugeteilt. Im 1-JahresVerlauf hatten die Patienten des Clopidogrel-Arms weniger Blutungskomplikationen ohne eine Zunahme des kombinierten Endpunkts von Gesamtsterblichkeit, Myokardinfarkt, Schlaganfall und Blutung. Die Ergebnisse der POPularAGE-Studie werden durch eine Subanalyse des SWEDEHEART(Swedish Websystem for Enhancement and Development of Evidence-based care in Heart disease Evaluated According to Recommended Therapies)-Registers bestätigt [20]. In dieser Analyse war Ticagrelor bei alten Patienten ( $\geq 80$ Jahre) im Vergleich zu Clopidogrel mit einem höheren Risiko von Blutungen und einer erhöhten Sterblichkeit verbunden. Auch eine Subanalyse der ISAR-REACT-5-Studie spricht für eine abgeschwächte $\mathrm{P} 2 \mathrm{Y}_{12}$-Inhibition bei älteren Patienten [21]. In dieser Studie wurde die Dosis von Prasugrel bei den $\geq 75$-Jährigen auf $5 \mathrm{mg}$ reduziert. Im Vergleich zur vollen Dosis von Ticagrelor ergaben sich keine erkennbare Einschränkung der Wirksamkeit, jedoch ein Trend zugunsten von weniger schweren Blutungen sowie eine signifikante Reduktion der Gesamtblutungsrate.

Deeskalation. Während sich ältere Studien vorwiegend mit der Intensivierung der antithrombozytären Therapie beschäftigen, steht in jüngster Zeit das Konzept der Deeskalation mit Abschwächung der Thrombozyteninhibition im Fokus des Interesses. Schon die ESCLeitlinie zur Myokardrevaskularisation hatte die Deeskalation der antithrombozytären Therapie als Option v. a. für Patienten mit erhöhtem Blutungsrisiko erwähnt. Diese Empfehlung wird in der neuen Leitlinie zu NSTE-ACS aufgegriffen. Unterstützend erschien hierzu 2020 die randomisierte TICO(Ticagrelor Monotherapy After 3 Months in the Patients Treated With New Generation Sirolimus-eluting Stent for Acute Coronary Syndrome)-Studie [22]. In dieser Studie wurden Patienten mit akutem Koronarsyndrom, die initial mit Ticagrelor und Azetylsalizylsäure (ASS) 
Hier steht eine Anzeige.

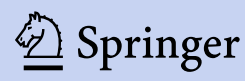


behandelt wurden, nach 3 Monaten auf eine Fortführung der dualen antithrombozytären Therapie (DAPT) oder eine Ticagrelor-Monotherapie randomisiert. Der Wechsel auf die TicagrelorMonotherapie erbrachte eine geringe, aber statistisch signifikante Reduktion des kombinierten Endpunkts von Blutung und kardiovaskulären Ereignissen nach 1 Jahr. Da die Studie vorzeitig abgebrochen wurde, nachdem $3056 \mathrm{~Pa}$ tienten eingeschlossen waren, sind die Ergebnisse vorsichtig zu interpretieren. Konsistente Ergebnisse wurden auch in der TWILIGHT-ACS-Subanalyse der TWILIGHT(Ticagrelor With Aspirin or Alone in High-Risk Patients After Coronary Intervention)-Studie erzielt [23]. Hier erbrachte das Absetzen von ASS nach 3 Monaten DAPT mit Ticagrelor eine signifikante und klinisch relevante Reduktion der Blutungsereignisse, ohne das ischämische Risiko zu erhöhen.

Die HOST-REDUCE-POLYTECHACS-Studie untersuchte die Deeskalation der DAPT bei Patienten, die initial mit Prasugrel $10 \mathrm{mg}$ plus ASS behandelt wurden [24]; 3429 Patienten wurden randomisiert entweder der Deeskalationsgruppe mit Reduktion der PrasugrelDosis auf $5 \mathrm{mg}$ nach 1 Monat oder der konventionellen Gruppe zugeteilt. Es ergab sich ein positiver Nettoeffekt zugunsten der Deeskalation (Gesamtsterblichkeit, nichtfataler Myokardinfarkt, Stentthrombose, erneute Revaskularisation, Schlaganfall und Blutungsereignisse) nach $1 \mathrm{Jahr}$, die von einer Reduktion der Blutungskomplikationen getrieben war.

Sekundärprävention. Aufgrund unterschiedlicher Studien hatte bereits die 2019 vorgestellte ESC-Leitlinie zu chronischen Koronarsyndromen empfohlen, im Rahmen der Sekundärprävention bei Patienten, bei denen das ischämische Risiko das Blutungsrisiko überwiegt, langfristig zusätzlich zu ASS eine zweite antithrombotische Substanz zu verordnen. Als Optionen wurden niedrig dosiertes Rivaroxaban, Clopidogrel, Ticagrelor oder Prasugrel genannt. Die neue ESC-Leitlinie zu NSTE-ACS greift diese Empfehlung auf. Unterstützend wurde hierzu 2020 eine neue Subanalyse der PEGASUS-TIMI-54(Prevention of Cardiovascular Events in Patients with Prior Heart Attack Using Ticagrelor Compared to Placebo on a Background of Aspirin-Thrombolysis in Myocardial Infarction 54)-Studie publiziert [25]. Diese zeigt, dass die langfristige Verordnung von Ticagrelor zusätzlich zu ASS im Vergleich zu ASS alleine auch bei Patienten mit vorausgegangenem Myokardinfarkt ohne PCI zu einer Reduktion des ischämischen Risikos führt, wenngleich auf Kosten eines erhöhten Blutungsrisikos.

\section{Fazit für die Praxis}

- Entsprechend der ISCHEMIA-Studie ist bei Patienten mit chronischem Koronarsyndrom ohne kürzlich vorausgegangenes akutes Koronarsyndrom (ACS), Herzinsuffizienz oder Hauptstammstenose die Indikation zur Myokardrevaskularisation kritisch zu prüfen. Ein engmaschiges Monitoring medikamentös behandelter Patienten ist notwendig: bei Persistenz der Beschwerden führt die Myokardrevaskularisation zu Lebensqualitätsverbesserung und Symptomkontrolle.

- Altersunabhängig wird bei allen Patienten mit NSTE-ACS und HochrisikoCharakteristika eine früh-invasive Behandlung angestrebt. Dabei erfolgt keine Vorbehandlung mit P2Y12Antagonisten, solange die Indikation zur Intervention nicht geklärt ist. Außer im kardiogenen Schock wird bei NSTE-ACS oder STEMI mit Mehrgefäß-Erkrankung eine komplette Revaskularisation angestrebt.

- Die chronische DAPT sollte in Abwägung des ischämischen und hämorrhagischen Risikos individualisiert werden. Neben den etablierten Therapieregimen umfasst dies einerseits das Absetzen von ASS oder die Dosisreduktion von Prasugrel nach 3 Monaten sowie die primäre Wahl von Clopidogrel und andererseits die langfristige Therapie mit zwei antithrombotischer Substanzen.

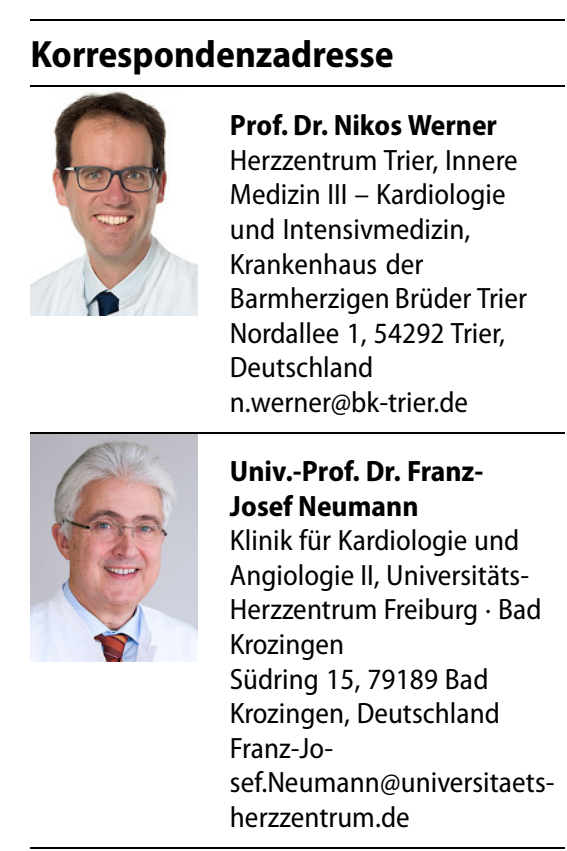

\section{Einhaltung ethischer Richtlinien}

Interessenkonflikt. N. Werner berichtet, Vortragshonorare erhalten zu haben von Abiomed, Bayer Healthcare, Boehringer Ingelheim, Boston Scientific, Daiichi Sankyo, Edwards Lifesciences, Medtronic, Novartis, Pfizer, Shockwave Medical und Terumo; finanzielle Studienunterstützung von Abiomed und Shockwave Medical. F.-J. Neumann berichtet Vortragshonorare an seine Institution von Amgen, Bayer Healthcare, Biotronic, Boehringer Ingelheim, Boston Scientific, Daiichi Sankyo, Edwards Lifesciences, Ferrer, Pfizer, Novartis; Beratungshonorare an seine Institution von Boehringer Ingelheim und Novartis; finanzielle Studienunterstützung von Bayer Healthcare, Boston Scientific, Biotronic, Edwards Lifesciences, GlaxoSmithKline, Medtronic, Pfizer.

Für diesen Beitrag wurden von den Autoren keine Studien an Menschen oder Tieren durchgeführt. Für die aufgeführten Studien gelten die jeweils dort angegebenen ethischen Richtlinien.

\section{Literatur}

1. Knuuti J, Wijns W, Saraste A, Capodanno D, Barbato E, Funck-Brentano C, Prescott E, Storey RF, Deaton C, Cuisset T, Agewall S, Dickstein K, Edvardsen T, Escaned J, Gersh BJ, Svitil P, Gilard M, HasdaiD, Hatala R, Mahfoud F,MasipJ, MunerettoC, Valgimigli M, Achenbach S, Bax JJ, ESC Scientific Document Group (2020) 2019 ESC Guidelines for the diagnosis and management of chronic coronary syndromes. Eur Heart J 41(3):407-477. https://doi.org/10.1093/eurheartj/ehz425

2. Maron DJ, Hochman JS, Reynolds HR, Bangalore S, O'Brien SM, Boden WE, Chaitman BR, Senior R, López-Sendón J, Alexander KP, Lopes RD, Shaw LJ, Berger JS, Newman JD, Sidhu MS, Goodman SG, Ruzyllo W, Gosselin G, Maggioni AP, White HD, Bhargava B, Min JK, Mancini GBJ, Berman DS, Picard MH, Kwong RY, Ali ZA, Mark DB, Sper- 
tus JA, Krishnan MN, Elghamaz A, Moorthy N, Hueb WA, Demkow M, Mavromatis K, Bockeria O, Peteiro J, Miller TD, Szwed H, Doerr R, Keltai M, Selvanayagam JB, Steg PG, Held C, Kohsaka S, Mavromichalis $S$, Kirby R, Jeffries NO, Harrell FE Jr, Rockhold FW, Broderick S, Ferguson TB Jr, Williams DO, Harrington RA, Stone GW, Rosenberg $Y$, ISCHEMIA Research Group.Maron DJ et al (2020) Initial invasive or conservative strategy for stable coronarydisease. NEnglJMed 382(15):1395-1407. https://doi.org/10.1056/NEJMoa1915922

3. Chaitman BR, Alexander KP, Cyr DD, Berger JS, Reynolds HR, Bangalore S, Boden WE, Lopes RD, Demkow M, Perna GP, Riezebos RK, McFalls EO, Banerjee S, Bagai A, Gosselin G, O'Brien SM, Rockhold FW, Waters DD, Thygesen KA, Stone GW White HD, Maron DJ, Hochman JS, ISCHEMIA Research Group (2020) Circulation. https://doi. org/10.1161/CIRCULATIONAHA.120.047987

4. Collet JP, Thiele H, Barbato E, Barthelemy O, Bauersachs J, Bhatt DL, Dendale P, Dorobantu M, Edvardsen T, Folliguet T, Gale CP, Gilard M, Jobs A, Juni $P$, Lambrinou E, Lewis BS, Mehilli J, Meliga E, Merkely B, Mueller C, Roffi M, Rutten FH, Sibbing D, Siontis GCM, Group ESCSD (2020) 2020 ESC Guidelines for the management of acute coronary syndromes in patients presenting without persistent ST-segment elevation. Eur Heart J ehaa575. https://doi.org/10.1093/eurheartj/ehaa575. (Online ahead of print.Eur Heart J. 2020. PMID: 32860058)

5. Kaura A, Sterne JAC, Trickey A, Abbott S, Mulla A, Glampson B, Panoulas V, Davies J, Woods K, Omigie J, Shah AD, Channon KM, Weber JN, Thursz MR, Elliott $\mathrm{P}$, Hemingway $\mathrm{H}$, Williams $\mathrm{B}$, Asselbergs FW, O'Sullivan M, Lord GM, Melikian N Johnson T, Francis DP, Shah AM, Perera D, Kharbanda R, Patel RS, Mayet J (2020) Invasive versus non-invasive management of older patients with non-ST elevation myocardial infarction (SENIOR-NSTEMI): a cohort study based on routine clinical data. Lancet 396(10251):623-634

6. Chacko L, Howard JP, Rajkumar C, Nowbar AN, Kane C, Mahdi D, Foley M, Shun-Shin M, Cole G, Sen S, Al-Lamee R, Francis DP, Ahmad Y (2020) Effects of percutaneous coronary intervention on death and myocardial infarction stratified by stable and unstable coronary artery disease: a meta-analysis of randomized controlled trials. Circ Cardiovasc Qual Outcomes 13(2):e6363

7. Linde JJ, Kelbaek H, Hansen TF, Sigvardsen PE, Torp-Pedersen C, Bech J, Heitmann M, Nielsen OW, Hofsten D, Kuhl JT, Raymond IE, Kristiansen OP, Svendsen IH, Vall-Lamora MHD, Kragelund C, de Knegt M, Hove JD, Jorgensen T, Fornitz GG, Steffensen R, Jurlander B, Abdulla J, Lyngbaek S, Elming H, Therkelsen SK, Jorgensen E, Klovgaard L, Bang LE, Hansen PR, HelqvistS, Galatius S, Pedersen F, AbildgaardU, Clemmensen P, Saunamaki K, Holmvang L, Engstrom T, Gislason G, Kober LV, Kofoed KF (2020) Coronary $\mathrm{CT}$ angiography in patients with non-STsegment elevation acute coronary syndrome. J Am Coll Cardiol 75(5):453-463

8. Lemkes JS, Janssens GN, van der Hoeven NW, Jewbali LSD, Dubois EA, Meuwissen MM, Rijpstra TA, Bosker HA, Blans MJ, Bleeker GB, Baak RR, Vlachojannis GJ, Eikemans BJW, van der Harst $P$ van der Horst ICC, Voskuil M, van der Heijden JJ, Beishuizen A, Stoel M, Camaro C, van der Hoeven H, Henriques JP, Vlaar APJ, Vink MA, van den Bogaard B, Heestermans T, de Ruijter W, Delnoij TSR, Crijns $\mathrm{H}$, Jessurun GAJ, Oemrawsingh PV, Gosselink MTM, Plomp K, Magro M, Elbers PWG, Spoormans EM, van de Ven PM, Oudemans-van Straaten HM, van
Royen N (2020) Coronaryangiography after cardiac arrest without ST segment elevation: one-year outcomes of the COACT randomized clinical trial. JAMA Cardiol 5(12):1-8. https://doi.org/10.1001/ jamacardio.2020.3670 (Online ahead of print)

9. Chew DP, Hyun K, Morton E, Horsfall M, Hillis GS, Chow CK, Quinn S, D'Souza M, Yan AT, Gale CP, Goodman SG, Fox K, Brieger D (2020) Objective risk assessment vs standard care for acute coronary syndromes: a randomized clinical trial. JAMA Cardiol e206314. https://doi.org/10. 1001/jamacardio.2020.6314 (Online ahead of print. JAMA Cardiol. 2020.PMID:33295965)

10. Bainey KR, Engstrom T, Smits PC, Gershlick AH, James SK, Storey RF, Wood DA, Mehran R, Cairns JA, Mehta SR (2020) Complete vs culprit-lesiononly revascularization for ST-segment elevation myocardial infarction: a systematic review and meta-analysis. JAMA Cardiol 5(8):1-9. https://doi. org/10.1001/jamacardio.2020.1251 (Online ahead of print. JAMA Cardiol. 2020.PMID: 32432651)

11. Pavasini $R$, Biscaglia $S$, Barbato $E$, Tebaldi $M$, Dudek D, Escaned J, Casella G, Santarelli A, Guiducci V, Gutierrez-Ibanes E, Di Pasquale G, Politi L, Saglietto A, D'Ascenzo F, Campo G (2020)Complete revascularization reduces cardiovascular death in patients with ST-segment elevation myocardial infarction and multivessel disease: systematic review and meta-analysis of randomized clinical trials. Eur Heart J 41(42):4103-4110

12. Sheth T, Pinilla-Echeverri N, Moreno R, Wang J, Wood DA, Storey RF, Mehran R, Bainey KR, Bossard M, Bangalore S, Schwalm JD, Velianou JL, Valettas N, Sibbald M, Rodes-Cabau J, Ducas J, Cohen EA, Bagai A, Rinfret S, Newby DE, Feldman L, Laster SB, Lang IM, Mills JD, Cairns JA, Mehta SR (2020) Nonculprit lesion severity and outcome of revascularization in patients with STEMI and multivessel coronary disease. J Am Coll Cardiol 76(11):1277-1286

13. Khera R, Secemsky EA, Wang Y, Desai NR, Krumholz HM, Maddox TM, Shunk KA, Virani SS, Bhatt DL, Curtis J, Yeh RW (2020) Revascularization practices and outcomes in patients with multivessel coronary artery disease who presented with acute myocardial infarction and Cardiogenic shock in the US, 2009-2018. JAMA Intern Med 180(10):1317-1327

14. Kim HS, Kang J, Hwang D, Han JK, Yang HM, Kang HJ, Koo BK, Kim SY, Park KH, Rha SW, Shin WY, Lim HS, Park K, Park KW, Investigators H-R-P-ARCT (2020) Durable polymer versus biodegradable polymer drug-eluting stents after percutaneous coronary intervention in patients with acute coronary syndrome: the HOST-REDUCEPOLYTECH-ACS trial. Circulation. https://doi.org/ 10.1161/CIRCULATIONAHA.120.051700 (Online ahead of print)

15. Valina C, Neumann FJ, Menichelli M, Mayer K, Wohrle J, Bernlochner I, Aytekin A, Richardt G, Witzenbichler B, Sibbing D, Cassese S, Angiolillo DJ, Kufner S, Liebetrau C, Hamm CW, Xhepa E, Hapfelmeier A, Sager HB, Wustrow I, Joner $M$, Trenk D, Laugwitz KL, Schunkert H, Schupke S, Kastrati A (2020) Ticagrelor or prasugrel in patients with non-ST-segment elevation acute coronary syndromes. J Am Coll Cardiol 76(21):2436-2446

16. Aytekin A, Ndrepepa G, Neumann FJ, Menichelli M, Mayer K, Wohrle J, Bernlochner I, Lahu S, Richardt G, Witzenbichler B, Sibbing D, Cassese $S$ Angiolillo DJ, Valina C, Kufner S, Liebetrau C, Hamm CW, Xhepa E, Hapfelmeier A, Sager HB, Wustrow I, Joner M, TrenkD, Fusaro M, Laugwitz KL, SchunkertH, SchupkeS, Kastrati A (2020) Ticagrelor or prasugrel in patients with ST-segmentelevation myocardial infarction undergoing primary percutaneous coronary intervention. Circulation 142(24):2329-2337

17. Turgeon RD, Koshman SL, Youngson E, Har B, Wilton SB, James MT, Graham MM (2020) Association of ticagrelor vs clopidogrel with major adverse coronary events in patients with acute coronary syndrome undergoing percutaneous coronary intervention. JAMA Intern Med 180(3):420-428

18. You SC, Rho Y, Bikdeli B, Kim J, Siapos A, Weaver J, Londhe A, Cho J, Park J, Schuemie M, Suchard MA, Madigan D, Hripcsak G, Gupta A, Reich CG, Ryan PB Park RW, Krumholz HM (2020) Association of ticagrelor vs clopidogrel with net adverse clinical events in patients with acute coronary syndrome undergoing percutaneous coronary intervention. JAMA 324(16):1640-1650

19. Gimbel M, Qaderdan K, Willemsen L, Hermanides $\mathrm{R}$, Bergmeijer T, de Vrey E, Heestermans T, Tjon Joe Gin M, Waalewijn R, Hofma S, den Hartog F, Jukema W, von Birgelen C, Voskuil M, Kelder J, Deneer V, Ten Berg J. Clopidogrel versus ticagrelor or prasugrel in patients aged 70 years or older with non-STelevation acute coronary syndrome (POPular AGE): the randomised, open-label, non-inferiority trial. Lancet 2020;395(10233):1374-1381.

20. Szummer K, Montez-Rath ME, Alfredsson J, Erlinge D, Lindahl B, Hofmann R, Ravn-Fischer A, Svensson P, Jernberg T (2020) Comparison between ticagrelor and clopidogrel in elderly patients with an acute coronary syndrome: insights from the SWEDEHEART registry. Circulation 142(18):1700-1708

21. Menichelli M, Neumann FJ, Ndrepepa G, Mayer K, WohrleJ,Bernlochnerl,RichardtG, WitzenbichlerB, Sibbing D, Gewalt $S$, Angiolillo DJ, Lahu $S$, Hamm CW, Hapfelmeier A, Trenk D, Laugwitz KL, Schunkert H, Schupke S, Kastrati A (2020) Age-and weight-adapted dose of prasugrel versus standard dose of ticagrelor in patients with acute coronary syndromes : results from a randomized trial. Ann Intern Med 173(6):436-444

22. Kim BK, Hong SJ, Cho YH, Yun KH, Kim YH, Suh Y, Cho JY, Her AY, Cho S, Jeon DW, Yoo SY, Cho DK, Hong BK, Kwon H, Ahn CM, Shin DH, Nam CM, Kim JS, Ko YG, Choi D, Hong MK, Jang $Y$, Investigators T (2020) Effect of ticagrelor monotherapy vs ticagrelor with aspirin on major bleeding and cardiovascular events in patients with acute coronary syndrome: the TICO randomized clinical trial.JAMA323(23):2407-2416

23. Baber U, Dangas G, Angiolillo DJ, Cohen DJ, Sharma SK, Nicolas J, Briguori C, Cha JY, Collier T, Dudek D, Dzavik V, Escaned J, Gil R, Gurbel P, Hamm CW, Henry T, Huber K, Kastrati A, Kaul U, Kornowski R, Krucoff M, Kunadian V, Marx SO, Mehta S, Moliterno D, Ohman EM, Oldroyd K, Sardella G, Sartori S, Shlofmitz R, Steg PG, Weisz G, Witzenbichler B, Han YL, Pocock S, Gibson CM, Mehran R (2020) Ticagrelor alone vs. ticagrelor plus aspirin following percutaneous coronary intervention in patients with non-ST-segment elevation acute coronary syndromes: TWILIGHTACS. Eur Heart J 41(37):3533-3545

24. Kim HS, Kang J, Hwang D, Han JK, Yang HM, Kang HJ, Koo BK, Rhew JY, Chun KJ, Lim YH, Bong JM, Bae JW, Lee BK, Park KW, Investigators H-R-P-A (2020) Prasugrel-based de-escalation of dual antiplatelet therapy after percutaneous coronary intervention in patients with acute coronary syndrome (HOST-REDUCE-POLYTECH- 
ACS): an open-label, multicentre, non-inferiority randomised trial. Lancet 396(10257):1079-1089

25. Furtado RHM, Nicolau JC, Magnani G, Im K, Bhatt DL, Storey RF, Steg PG, Spinar J, Budaj A, Kontny F, Corbalan R, Kiss RG, Abola MT, Johanson P, Jensen EC, Braunwald E, Sabatine MS, Bonaca MP (2020) Long-term ticagrelor for secondary prevention in patients with prior myocardial infarction and no history of coronary stenting: insights from PEGASUS-TIMI 54. Eur Heart J 41(17):1625-1632

\section{Diastolische Herzschwäche: Diagnostik mit Echtzeit-MRT kann Alternative zur Herzkatheter-Untersuchung sein}

Bei einer diastolischen Herzschwäche, die auch Heart failure with preserved ejecton fraction (HFpEF) genannt wird, bleibt die Pumpkraft des Herzens erhalten, aber die linke Herzkammer ist steif und füllt sich nicht ausreichend mit sauerstoffreichem Blut. Die Patienten leiden unter Atemnot, Wassereinlagerungen und sind körperlich zunehmend weniger leistungsfähig. Mit nichtinvasiven Methoden, wie einer Ultraschalluntersuchung, war die HFpEF bisher schwer zu diagnostizieren und wurde oft erst spät erkannt.

In der Studie HFpEF-stress-DZHK17 konnten die Wissenschaftler um Studienleiter Professor Dr. Andreas Schuster, Klinik für Kardiologie und Pneumologie der Universitätsmedizin Göttingen (UMG), nun zeigen, dass die diastolische Herzschwäche mithilfe einer neuen nicht-invasiven Echtzeit-MRTTechnologie präzise diagnostiziert und somit eine Herzkatheter-Untersuchung zukünftig möglicherweise vermieden werden kann.

Professor Dr. Martin Uecker, Institut für Diagnostische und Interventionelle Radiologie der UMG, sowie Dr. Shuo Zhang und Professor Dr. Jens Frahm vom Max-Planck-Institut für biophysikalische Chemie in Göttingen entwickelten die neue MRT-Technologie. Sie erlaubt es, MRT-Messungen am Herzen unter Belastung durchzuführen. Denn die Patienten können bei der MRT-Untersuchung weiteratmen und müssen nicht, wie bisher, währenddessen den Atem anhalten.

Eingeschränkte Herzfunktion wird unter Belastung deutlich

Bei der am Göttingen Campus entwickelten Methode ist auf der Untersuchungsliege eine Art Hometrainer installiert. Die Besonderheit liegt in den nicht-magnetischen Bauteilen des MRT Ergometers, die dessen Einsatz im Magnetfeld des Magnetresonanztomographen ermöglichen. Die Patienten fahren liegend Fahrrad, dabei vermisst das MRTGerät ihr Herz. Die Bilder können die Ärzte während der Untersuchung auf einem Bildschirm verfolgen und so genau beurteilen, wie gut das Pumporgan des Patienten funktioniert. „Wir sehen im MRT, wie das Herz schlägt, wie es sich füllt und wieder entleert", erklärt der Erstautor der Studie, Privatdozent Dr. Sören Backhaus, Klinik für Kardiologie und Pneumologie der UMG. „Mit dem MRT können wir daher direkt die krankmachende Veränderung am Herzen messen und nicht nur die Konsequenzen beurteilen." Bei Patienten mit HFpEF ist dabei zu sehen, dass die Leistung des linken Vorhofs des Herzens eingeschränkt ist. Das ist die erste Herzkammer, die das mit Sauerstoff beladene Blut nach der Lunge erreicht. „Diese eingeschränkte Funktion wird aber nur deutlich, wenn die Patienten sich bewegen und damit Herz und Kreislauf belasten", so Backhaus.

\section{Multizentrische Studien geplant}

Bei dem bisherigen Goldstandard der Herzuntersuchung wird ein Katheter über die Lungenarterie in das Herz geschoben und misst die Veränderung des Lungendrucks, während sich die Patienten bewegen. Bei Patienten mit einer diastolischen Herzschwäche staut sich unter Belastung das Blut in die Lunge zurück, sodass der Lungendruck zunimmt. Die Untersuchung mit dem Herzkatheter ist zwar sehr genau, aber teuer, für die Patienten belastend und auch nicht einfach umzusetzen, da sie sich bewegen müssen, während ein Katheter in ihrem Herzen liegt.

Mit der DZHK-Studie haben die Göttinger Wissenschaftler die neue MRT-Untersuchung validiert und bewiesen, dass sie für die Diagnose der HFpEF sehr gut funktioniert und die Katheteruntersuchung so möglicherweise vermieden werden kann. Bevor das Verfahren routinemäßig in der Diagnostik eingesetzt werden kann, sind aber noch größere Studien notwendig. „Bei der FahrradErgometrie Echtzeit MRT handelt es sich um ein komplett neues diagnostisches Verfahren für Patienten mit diastolischer Herzschwäche. Als Nächstes planen wir eine Studie, an der sich mehrere Zentren beteiligen, um zu überprüfen, ob die Methode für die Patienten vorteilhaft ist", so Schuster

Quelle: www.dzhk.de 